\title{
The effects of interaction with the device described by procedural text on recall, true/false, and task performance
}

\author{
VIRGINIA A. DIEHL \\ Western Illinois University, Macomb, Illinois \\ and \\ CAROL BERGFELD MILLS \\ Goucher College, Towson, Maryland
}

\begin{abstract}
In two experiments, subjects interacted to different extents with relevant devices while reading two complex multistep procedural texts and were then tested with task performance time, true/false, and recall measures. While reading, subjects performed the task (read and do), saw the experimenter perform the task (read and see experimenter do), imagined doing the task (read and imagine), looked at the device while reading (read and see), or only read (read only). Van Dijk and Kintsch's (1983) text representation theory led to the prediction that exposure to the task device (in the read-and-do, read-and-see, and read-and-see-experimenter-do conditions) would lead to the development of a stronger situation model and therefore faster task performance, whereas the read-only and read-andsee conditions would lead to a better textbase, and therefore better performance on the true/false and recall tasks. Paivio's (1991) dual coding theory led to the opposite prediction for recall. The results supported the text representation theory with task performance and recall. The read-and-see condition produced consistently good performance on the true/false measure. Amount of text study time contributed to recall performance. These findings support the notion that information available while reading leads to differential development of representations in memory, which, in turn, causes differences in performance on various measures.
\end{abstract}

How do readers' interactions with the device described by a procedural text affect memory and comprehension of the text and the ability to perform the task described by a text? To answer this question, in two experiments we had readers interact with procedural texts to different extents and in different ways. For example, they performed the task while reading, only looked at the device described by the text while reading, or only read the text.

Van Dijk and Kintsch (1983) proposed that as a reader reads, propositional and situational representations are formed in long-term memory. The propositional representation (or textbase) holds the text's meaning, and the situational model represents the situation described by the text. Van Dijk and Kintsch have proposed that these representations can be developed differentially while reading and that the extent to which they are developed leads to differences in performance on various measures. For

Experiment 1 was supported in part by a grant from the U.S. Army Human Engineering Laboratory (Contract DAAA 15-87-K000-4) to American Institutes for Research. We gratefully acknowledge the assistance of Corliss Cartier, Jill Crockett, Teresa Lachman-Jones, Sharon Metro, Lien-chong Mou, Deborah Quick, and Stacey Swain in running the subjects and/or scoring the data. We also thank Danielle McNamara, Ruth Maki, and Peter Dixon for their valuable comments on an earlier draft of this manuscript. Requests for reprints may be addressed to Virginia A. Diehl, Psychology Department, Western Illinois University, Macomb, IL 61455. example, the development of a propositional representation will lead to better recall, while development of a representation of the situation described by the text will lead to more accurate inferencing (Perrig \& Kintsch, 1985).

One variable that seems to influence the differential development of different representations of a text is the reader's goal. Schmalhofer and Glavanov (1986) had subjects read a programmer's manual either to summarize it or to acquire knowledge. Summarization subjects spent more time reading high-level information, and their recognition performance reflected the development of both strong propositional and situational representations. Knowledge-acquisition subjects spent more time reading information that provided substantive information about the programming language, and their recognition performance revealed a strong situational representation. This suggests that one's focus during learning affects the strength of the representations that are formed, which, in turn, influences recognition performance.

Mills, Diehl, Birkmire, and Mou (1995) investigated the effect of a reader's goal when reading a procedure (to perform the task or to recall the text) on recall, task performance, and reading rate. The task-performance subjects spent less time reading the parts of the text that were unimportant for task performance than did the textrecall subjects. The task-performance subjects performed the task better, while the text-recall subjects recalled the 
text better. These findings suggest that a reader's goal influences the time spent on different types of text information, and this affects the reader's internal representations of the task and text, which, in turn, produce differences in recall and task performance.

A study by Mannes and Kintsch (1987) investigated how two types of advance organizers (consistent or inconsistent with the organization of the text) influenced recall, recognition, and problem solving. They predicted that a consistent organizer would lead to a stronger propositional representation, and this would be reflected in better recall. An inconsistent organizer, on the other hand, would lead to a better developed situational representation, which would result in more accurate inferencing on recognition items and better problem-solving performance. These predictions were supported, confirming the notion that memory and problem-solving performance are affected differently, depending on the supplementary information presented during learning. It appears that recall performance relies primarily on a propositional structure and recognition task inferencing and problem solving depend more on a situational representation.

How is a situation model developed in the course of reading a text? According to van Dijk and Kintsch (1983), the situational model is a representation that is built through experience. If we have a single experience, our situation model will be specific to that experience. When we read a text, we activate situation models we think are relevant, to use for comprehending the text. We also add to or revise our situation models as we read; this is the process of updating. As we read, supplementary information is often provided to help us understand the text. This could include pictures, diagrams, or objects. In the case of procedural text, we might have available the actual device to which the text refers. The availability of such related information will lead to a better situational model of the text. Using a procedural text effectively can be seen as a problem-solving task (a word problem). One must figure out the correspondence between the text, the device the text is referring to, and the task itself. If this is done effectively, then an adequate situation model is constructed. The knowledge or environmental cues one has available to apply will influence the quality of the inferences that are generated, and these, in turn, will affect the quality of the situational representation.

According to the text representation theory, performance on a particular type of task will be affected by the quality of a particular memory representation. It is possible to have an adequate text representation and an inadequate situational representation, or vice versa (Kintsch, 1994). In the present study, we measured recall, true/ false, and task performance. While all of these tasks can be partially accomplished using memory for either the text or the situation described by the text, recall should be influenced most by the propositional representation, particularly when the delay between reading and recall is short (McNamara, Kintsch, Songer, \& Kintsch, in press), as it was in the present study. The true/false task used in this study was very text-dependent. Like the recall task, a good propositional representation should lead to good performance on this test. On the other hand, problemsolving tests, like task performance, should be most affected by the situational representation (Mannes \& Kintsch, 1987), because one must understand the procedure in order to do the task successfully.

The extent to which a particular representation develops while reading depends, in part, on the type of additional information presented to the reader. In the present studies, we manipulated the type of additional information available to the subjects. In Experiment 1, subjects only read the text (read only), saw the device described by the text while reading (read and see), or did the task while reading (read and do). The tasks used were complex multistep procedures, yet their substeps were quite straightforward and not totally unfamiliar to most people.

The results of both Schmalhofer and Glavanov (1986) and Mills et al. (1995) suggest that readers who read with the purpose of understanding the task described by the text focus more on the parts of the text that describe the task. On the other hand, readers who read with the purpose of recalling the text allocate similar processing resources to all parts of the text, and longer reading time leads to better recall (Mills et al., 1995). The present readand-do condition should lead the reader to focus on the task and therefore a good situational model will be developed. With total amount of processing time held constant, having to do the task while reading will mean less time to spend with the text (because doing the task takes more time than just looking at the device or only reading the text), therefore a poorer propositional representation will be developed. The read-only condition will lead to the development of a good propositional representation (because the reader focuses on the text) but will not lead to the development of a strong situational representation, because the device is not available for reference. The readand-see subjects should be able to update their situational model if they see the device while reading. Reading the text while looking at the device should make the text concrete (and therefore allow subjects to understand its meaning) without making the task the main focus. In other words, read-and-see subjects should be able to develop both an adequate situational and propositional representation. However, their situation model will not be as good as that of read-and-do subjects, who have done the task during encoding - that is, they have engaged in transfer-appropriate processing (e.g., Blaxton, 1989).

Therefore, the text representation theory leads to the predictions that the read-and-do subjects, because they have the best situational representation, will do better (i.e., perform faster) on the task performance measure than will the read-and-see subjects, who will perform better than will the read-only subjects. However, the read-only and read-and-see subjects will show better recall and true/false performance than will the read-anddo subjects, because more time with the text will lead to a better propositional representation.

The predictions of the text representation theory contrast with those of Paivio's (1991) dual coding theory. In 
addition to testing the text representation theory, we investigated how well the recall data fit the predictions of dual coding theory. According to Paivio, long-term memory contains two independent but interacting subsystems. One represents linguistic information (e.g., spoken or written), and the other represents nonlinguistic information (e.g., pictures or object manipulation). The implication of the subsystems' independence is that when the same information is coded both verbally and nonverbally, it will have greater mneumonic power than when only coded one way. In addition, according to this theory, if nonverbal information is processed in more than one sensorimotor modality (e.g., visual and proprioceptive), this will have additive effects in recall.

Previous research using recall as the outcome measure has provided support for this theory. Research on the effects of performing very simple procedures has shown that motoric enactment of to-be-remembered material improves memory for that material. Cohen (1981) found that when subjects were read a series of instructions for minitasks and then performed them (e.g., cross your fingers; put on the thimble), they were better able to recall the instructions than when the series of instructions was just read to them and was not performed.

Saltz and his associates (Saltz \& Dixon, 1982; Saltz \& Donnenwerth-Nolan, 1981) have studied the effect that motoric enactment of sentences has on sentence retention. Saltz and Donnenwerth-Nolan (1981) found that motoric enactment of sentences (e.g., The chef flipped the pancake; The workman dug a hole in the ground) produced better recall of the sentences than did just repeating the sentences out loud twice when subjects were later given the subject of the sentence to cue its recall. Furthermore, in their experiments in which competing tasks were used, the results suggested that motoric enactment leads to some type of motoric trace, whereas forming a visual image leads to an independent visual trace. Both of these traces appear to be relatively independent of a verbal trace.

A number of other studies have also shown that motoric enactment has a positive effect on memory. Blake, Austin, and Lowenstein (1987) showed that acting out sentences with toys facilitated preschool children's recall. Bäckman (1985) showed that older adults' free recall of subject-performed tasks was better when they actually performed them with the object (e.g., thimble for "put on the thimble") than when a procedure was presented with an appropriate object but they did not perform it. However, for younger adults, there was no difference in free recall between performing the procedures or not when the appropriate object was present.

Thus, the research with motoric enactment and subject-performed tasks suggests that coding the task motorically or visually, in addition to verbally, will facilitate later recall for the procedure. The text representation model might explain these results on the basis of the fact that understanding the task is the same as understanding the text with such simple procedures. The procedures used in the present research were different from those used in the previously reported research on dual coding.
The present texts were much longer, multistep procedures. To our knowledge, effects of task enactment on the recall of complex procedural texts have not been investigated. Since the present experiments used complex materials, task enactment, and a variety of dependent measures, the generality of these experiments' findings will be determined.

According to dual coding theory, the more codes that represent the information in memory, the better that information will be remembered. In the present experiments, read-and-do subjects had information coded in three ways. They had verbal, visual, and proprioceptive codes. Readand-see subjects developed a verbal and a visual code. Read-only subjects had a verbal code. While it is true that subjects in all conditions might have supplemented their text processing with imagery, those in the read-only condition could not have developed as accurate a visual representation as those in the other two conditions. Therefore, dual coding theory predicts that recall performance will be best in the read-and-do condition, second best in the readand-see condition, and worst in the read-only condition. The recall measure is the critical test of the text representation and dual coding theories, because they make clearly different predictions for this variable. The text representation theory predicts that read-and-do subjects will show the worst recall, whereas dual coding predicts that read-and-do subjects will produce the best recall.

Two additional conditions that have not yet been described were included in Experiment 1 to control for differences in how long it would take subjects to fulfill the requirements of each of the three conditions based on pilot work. Reading while doing the task (read and do) was expected to take longer than reading while looking at the device (read and see) or only reading (read only) and, as a consequence, subjects might do better in the read-and-do condition than in the other two conditions because they were exposed to the materials longer. Hence, the read-and-see-yoked and read-only-yoked conditions were introduced in which each of the subjects' exposure times were matched with a subject in the read-and-do condition. Even though the two yoked conditions were expected to take longer than the two corresponding unyoked conditions (which were called the read-and-seeunyoked and read-only-unyoked conditions), similar results might be expected in the yoked and unyoked conditions if the time working on the task was not as important as the type of representation that the reader constructs. Alternatively, more time available may make it possible to develop a more detailed representation of the text, resulting in better performance in the yoked conditions than in the unyoked conditions.

\section{EXPERIMENT 1}

\section{Design}

\section{Method}

Subjects participated in two experimental sessions. In the first session, they took a reading test and a mechanical abilities test that were used to match sets of 5 subjects. In the second session, each subject in a set read two experimental texts in one of five experi- 
mental conditions. In the read-and-do condition, the subjects performed the task while they read the text. In two other conditions (read-and-see-yoked and read-and-see-unyoked), the subjects looked at the objects while they read the text but did not perform the task. In the read-and-see-yoked condition, the subjects were given the same amount of time to read as the subjects in the readand-do condition in their subject set, whereas in the read-andsee-unyoked condition, they used the amount of time they wanted to read the text. In two other conditions (read-only-yoked and readonly-unyoked), the subjects were not allowed to see the objects needed for the task. In the read-only-yoked condition, they used the same amount of time as did the read-and-do subjects in their set, whereas in the read-only-unyoked condition, the subjects used the amount of time that they wanted. Immediately after reading the first text, each subject recalled the text, answered true/false questions about the text, and then performed the task while referring to the text. The same procedure was then repeated with the second text. The dependent measures were number of idea units correctly recalled, number of true/false questions correctly answered, and time to perform the task with the instructions available.

\section{Subjects}

The subjects were 50 students ( 44 females and 6 males) from a liberal arts college. The subjects were matched on the basis of their reading rates and mechanical abilities. There were 10 subjects in each of the five experimental conditions. Two additional subjects were run, but their data were not used because they could not perform one of the tasks. The subjects either received credit in their psychology class or were paid $\$ 10$ for participating.

\section{Materials}

For the first session, two standardized tests were used: the NelsonDenny Reading Test Version C (Brown, 1973) and the Mechanical Movements Test (Thurstone \& Jeffrey, 1984). From the NelsonDenny Reading Test, only the page of the test for determining reading rate was used. The Mechanical Movements Test was used in its entirety.

For the second session, two texts were used: "How to Use a Digital Timer Clock" (for Radio Shack clock/timer, Model 63-80) and "How to Make and Use a Spool Vehicle" (which is a set of instructions for making a children's toy, from Herbert, 1980). These texts will be called Clock and Spool, respectively. Clock consisted of 115 propositions, and Spool consisted of 119 propositions Clock and Spool are shown in the Appendix. Clock consisted of four subparts: how to read the time, how to set the time, how to replace the battery, and how to use the timer. Clock was written in short sentences with little elaboration or explanation of the steps. Spool consisted of the background of the toy, how to make the toy using a spool, rubber band, washer, tack, and pencil, and how to use the toy. There were longer sentences in this text, as well as explanations. These two structurally different procedural texts were selected in order to determine the generality of the obtained results.

The true/false tests consisted of 15 questions for each experimental text. Approximately half of the questions were true for each text, and half were false for each text. Most of the questions were fragments or slight variations of individual sentences from the text; others required a combination of the information from several sentences in order to answer them correctly. For example, questions that were based on individual text sentences for Clock and Spool, respectively, were: "Timer counts to 15 minutes" (true), "Normal display shows month and date" (false), and "A spool of any size may be used" (true), "The rubber band should be two times the length of the spool" (false). Questions that required integration for Clock and Spool, respectively, were: "Press D to change from one display to another" (true), "Press S and D at the same time to get the timer function" (false), and "The spool will keep going until the rubber band is unwound" (true), "You should put on the drag before the washer" (false).

The clock/timer along with two $3 \times 5$ in. cards with information about setting it were used for the performance task for the Clock. A spool, a matchstick, a tack, a pencil, a rubber band, and a metal washer were the objects used for the performance task for Spool.

A three-sentence practice text concerning the arrangement of a piece of paper, a paper clip, and a calculator was also used. A fivequestion true/false test about that text and the three objects described by the text were used to measure performance for the practice text.

A questionnaire that had three questions for each of the two experimental texts was used to gauge the subjects' prior familiarity with the tasks.

A countdown timer was used in the two yoked experimental conditions so that the subjects could monitor how much time they had remaining to read. A stopwatch was used to time the unyoked subjects' reading times and all subjects' performance times. A video camera, recorder, and monitor were used to record the subjects' task performance.

\section{Procedure}

The first session was run in groups of 1-7 subjects. In this session, the subjects were told that the purpose of the experiment was to "determine how people remember and use instructional texts to perform tasks." The mechanical aptitude test was administered following the instructions in the manual. The subjects then received one page from the Nelson-Denny test. The instructions were modified so that the subjects were timed for 2 min rather than 1 . At the end of $2 \mathrm{~min}$, they marked the line they were reading in order for the experimenter to determine their reading rate. They then continued reading and answered the comprehension questions. After all of the subjects were run in Session 1, they were matched as closely as possible in sets of 5 on the basis of mechanical aptitude scores and reading rates.

In Session 2, the subjects were run individually. One subject in each matched set of 5 subjects was randomly assigned to one of the five experimental conditions. All subjects read both texts, with half of the sets of subjects reading Clock first and half reading Spool first.

To simplify the presentation of the conditions, the procedures identical for all conditions will be described. The subjects in all conditions were asked to read the text all the way through twice, informing the experimenter at the end of the first reading. The experimenter recorded the "first-reading" time. After reading the text for the second time, the experimenter took away the text and any objects they had used while reading. The experimenter recorded the total reading time in the read-and-do and unyoked conditions. The subjects were then asked to recall the instructions in order and in complete sentences. The subjects were told that they did not need to recall the text word for word but should recall the main ideas or steps. When the subjects were finished recalling the text, the recall protocol was removed and the subjects were given the true/false questions to answer. Both the recall and the true/false questions were self-paced. After completing the true/false questions, the text and materials for performing the task were given to the subjects. The subjects were asked to perform the task described by the text while referring to the written text if necessary. We allowed them to refer to the text during task performance because this mimicks the way that people actually use procedural text. The subjects were videotaped so that their performance could be analyzed later, and the experimenter used a checklist to keep track of which critical steps the subject performed. The experimenter timed how long the subjects worked on the task until finished. The subjects were asked to refer back to the text if they had questions about the task.

The five experimental conditions differed from each other in terms of what the subjects did during the second time they read the 
text. During the second time the subjects read the text, the readand-do subjects performed the task. The read-and-see subjects looked at the objects for performing the task as they read about the task. The read-only subjects did not receive the objects for performing the task. A second way the experimental conditions differed was in terms of how much time the subjects had to read the text. The read-and-see-unyoked and the read-only-unyoked subjects set their own reading times and, the experimenter timed them. The read-and-see-yoked and the read-only-yoked subjects were yoked in total reading time to the read-and-do subjects in their matched set. These yoked subjects read through the text the first time at their own pace; however, on the second reading, they monitored how much time they had remaining to read on a countdown timer. The yoked subjects were instructed to reread the text until the timer went off.

For the Clock text, all subjects received two cards prior to the second reading. One card showed the order of the three buttons on the clock. The second card indicated the time and date to which the clock was to be set $(3: 17$ p.m. on $8 / 25)$. The subjects were told, when using the timer, to time $5 \mathrm{sec}$. They were given the cards so that all subjects would be thinking about doing the same thing during the second reading. Whenever the subjects were given the clock, it was set at the same time and date (12 p.m. on 12/12). The first set of 5 subjects attempted to perform the battery replacement section of the Clock instructions; however, because of numerous difficulties (e.g., losing screws and not being able to get the clock back together), later subjects were instructed not to perform this section of the instructions.

For the Spool text, the subjects in the read-and-see conditions were given the spool vehicle already put together.

After the subjects went through all of the tasks for the first text, they were given a short break and then performed the same tasks in the same condition for the second text. After completing both experimental texts, they received a questionnaire on their prior experience with the devices or with devices that were similar.

Prior to beginning the tasks with the two experimental texts, the subjects performed all of the tasks with the practice text in order to familiarize themselves with what they would be doing with the experimental texts.

\footnotetext{
Scoring

Any scores that fell more than 3 standard deviations away from the mean of that measure in the experiment were not used in the analyses. On the basis of this criterion, one Spool performance time measure for one of the read-only-unyoked subjects was eliminated. Because, as will be described later, the data were combined across the two texts for the planned comparisons, when a subject's score on a measure was eliminated for one of the texts, his or her score on that measure from the other text was excluded as well.

Recall. In previous research (Mills, Diehl, Birkmire, \& Mou, 1993), the texts were divided into propositions. For the present research, two judges independently scored whether each subject correctly recalled each proposition. A lenient set of criteria was used to score the recall data. If information from two propositions was combined during recall, both were counted as correct. The units did not have to be recalled in the order given in the text unless context was needed to disambiguate which unit was being recalled. After scoring the data, the judges met and resolved any differences. The percent agreement before discussion was $91 \%$ for Clock and $95 \%$ for Spool. Because some of the subjects did not recall the battery performance section of the text-presumably because they did not think they were supposed to recall it since they did not perform it - this section of the text was not scored for any of the subjects. The data were converted to the number of idea units (a larger unit that corresponds to phrases, based on the divisions made by Mills et al., 1993) correctly recalled by calculating the proportion of propositions correctly recalled in each idea unit. For
}

example, if an idea unit included three propositions and a subject only recalled one proposition correctly, then the subject recalled $33 \%$ of that idea unit. The number of idea units recalled was used in the analyses. The total number of idea units for Clock was 38 (without the section about replacing the battery) and for Spool was 34 .

True/false. The four questions about battery replacement from the Clock text were not scored; therefore, that text had a possible score of 11. True/false from the Spool text had a possible score of 15 . The number of correctly answered questions for each subject was used in the analyses.

Task performance. All the subjects whose data were used in the analyses completed the main steps of the tasks. Some subjects omitted a minor step or performed a minor error but thought they had completed the task correctly. The length of time that the subjects worked on the tasks (in hundredths of a minute) was used in the analyses, rather than number of errors. Performance time was used in this experiment rather than performance errors because, as we determined in another study (Mills et al., 1995), errors are not a very sensitive measure of task knowledge with this type of text.

The first set of 5 subjects performed the battery replacement section of the Clock text; however, due to problems with this part of the procedure, the remaining 45 subjects were instructed to not complete this step. The performance times for the first set of subjects were made comparable to the other subjects by subtracting the battery performance times out of their total performance times on the basis of the videotaped recordings of the perfor mance task.

The amount of time it took the subjects to put the rubber band through the hole in the spool was subtracted from the performance time for all subjects, on the basis of the videotaped recordings of the performance task, by a judge who was blind to experimental condition. This was done because (as will be reported later), in the second experiment, these times were subtracted from total performance time, and we wanted the two experiments to be comparable.

Familiarity. Familiarity scores were based on responses to the familiarity questionnaire. They were scored on a 4-point scale, with 0 representing no experience with the device and 3 representing exact or very similar experience. A familiarity score was determined for each of the two texts for each subject.

\section{Results and Discussion}

Before carrying out the comparisons of interest, several analyses were performed for each text separately to make sure that the groups were balanced with respect to familiarity with the tasks, first reading times, and the matching variables. Analyses of variance (ANOVAs) revealed no effect of condition on either familiarity with the task $(F \mathrm{~s}<1)$ or the amount of time the subjects took to read the texts the first time $\left(F_{\mathrm{S}}<1\right)$. There was no effect of condition on the matching variables (normed reading rate score from the Nelson-Denny Reading Test and number correct on the Mechanical Movements Test, $F_{\mathrm{S}}<1$ ).

To test for an effect of processing time on each of the dependent measures, a 2 (read-and-see or read-only) $\times$ 2 (yoked with read-and-do or unyoked) ANOVA was performed with each text separately. There was no significant effect of processing time and no interaction for the performance time and true/false measures $(F \mathrm{~s}<1)$. Therefore, we combined across the yoked and unyoked conditions for the performance time and true/false comparisons of interest. However, the recall measure showed a main effect of yoking. The subjects in the yoked conditions recalled more than did those in the unyoked con- 
ditions for both the Clock text [yoked $M=19.06$, and unyoked $M=14.25 ; F(1,36)=5.18, p<.05]$ and the Spool text [yoked $M=11.51$, and unyoked $M=8.97 ; F(1,36)=$ $4.06, p=.05]$; therefore, we did not combine the yoked and unyoked conditions for the recall measure.

For the following analyses only, we converted the true/ false and recall scores to proportions (because there were different possible scores for the two texts) and performed a three-way ANOVA on each of the dependent measures (true/false, recall, and performance time). The betweensubjects factors were text order and condition (read-anddo, read-and-see, and read-only), and the within-subjects factor was text. There were main effects of text for recall [mean proportion for Clock $=.42$, and mean proportion for Spool $=.29 ; F(1,40)=43.89, p<.001]$ and performance time [Clock $M=1.95$, and Spool $M=1.30$; $F(1,39)=16.50, p<.001]$. As hoped, for all three of the measures, there was no main effect of text order and none of the two- or three-way interactions were significant. The means and standard deviations for each condition and each measure are shown in Table 1. These data are shown combined across text order and text because these variables did not interact with each other or with condition.

To construct the predicted means from the two theories, we first determined the pattern of means that the theories would predict. This was done for all of the dependent measures for the text representation theory, but only for recall for dual coding. Equal intervals between different predicted means were assumed. Then, for each dependent measure, based on its grand mean and standard deviation across the entire experiment, we constructed the predicted means such that their grand mean and standard deviation closely matched those of the actual data. To test the goodness of fit of the theories, we compared the predicted means with the observed means using chi-square tests.

As can be seen in Figure 1, the read-and-do condition produced the best performance time score, the read-andsee condition was second, and the read-only condition was worst. However, only the read-and-do $(M=1.22)$ versus the read-only $(M=1.82)$ comparison was significant $[t(44)=-2.14, p<.05]$. The read-and-see condition did not differ from either the read-and-do condition or the read-only condition. The text representation theory accurately predicted the pattern of the performance time means $\left[\chi^{2}(2)=0.006, p<.01\right]$.

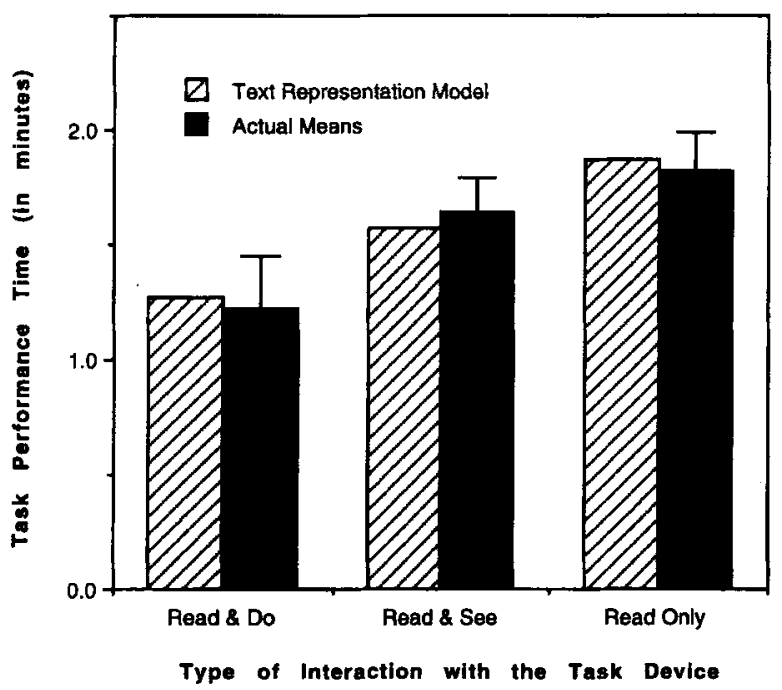

Figure 1. Text representation theory performance time predictions and observed means from Experiment 1.

That the read-and-do subjects performed the task faster than did the read-only subjects is consistent with the idea that the read-and-do condition led to a better situational representation than did the read-only condition. Because the read-and-do subjects did the task while reading, they had the advantage of transfer-appropriate processing. This should have led the read-and-do subjects to perform better than the read-and-see subjects, but this was not the case. The lack of a significant difference between the read-and-do condition and the readand-see condition suggests that the subjects in these conditions formed similar representations; however, this notion is weakened by the lack of difference between the read-and-see and read-only subjects. It is possible that the lack of significant differences between the read-andsee condition and the other conditions was due to the relatively small number of subjects used. To further explore this issue, more subjects were used in Experiment 2.

For the true/false measure, the text representation theory led to the prediction that the read-and-see and readonly subjects would answer more questions correctly than would the read-and-do subjects (see Figure 2). In fact, the read-and-do condition $(M=11.65)$ and readand-see condition $(M=11.62)$ appeared to be comparable and greater than the read-only condition $(M=10.78)$.

Table 1

Means From Experiment 1 Averaged Across Text and Order

\begin{tabular}{|c|c|c|c|c|c|c|c|c|c|c|}
\hline \multirow{3}{*}{$\begin{array}{l}\text { Dependent } \\
\text { Measure }\end{array}$} & \multicolumn{10}{|c|}{ Condition } \\
\hline & \multicolumn{2}{|c|}{ Read-and-Do } & \multicolumn{2}{|c|}{$\begin{array}{c}\text { Read-and-See- } \\
\text { Yoked }\end{array}$} & \multicolumn{2}{|c|}{$\begin{array}{c}\text { Read-and-See- } \\
\text { Unyoked }\end{array}$} & \multicolumn{2}{|c|}{ Read-Only-Yoked } & \multicolumn{2}{|c|}{$\begin{array}{c}\text { Read-Only- } \\
\text { Unyoked }\end{array}$} \\
\hline & $M$ & $S D$ & $M$ & $S D$ & $M$ & $S D$ & $M$ & $S D$ & $M$ & $S D$ \\
\hline Performance time & 1.22 & 0.73 & 1.65 & 0.77 & 1.63 & 0.60 & 1.86 & 0.74 & 1.78 & 0.77 \\
\hline True/false & 11.65 & 0.82 & 11.80 & 0.98 & 11.45 & 0.72 & 10.75 & 1.23 & 10.80 & 1.36 \\
\hline Recall & 11.61 & 4.89 & 16.03 & 4.30 & 12.93 & 4.43 & 14.55 & 5.65 & 10.29 & 4.54 \\
\hline
\end{tabular}

Note-Performance times are given in minutes. True/false values are expressed in terms of the number of questions correctly answered. Recall values are expressed in terms of the number of idea units correct. $n s=10$, except for read-only-unyoked performance time in which $n=9$. 
The good performance of the read-and-see subjects was as predicted; however, the performance of the subjects of the other two conditions was in the opposite direction from the predictions. The predictions of the text representation theory did not fit the data better than chance.

This pattern of results suggests that a well-developed textbase alone may not be sufficient for good true/false performance. However, this should not be the case, because the true/false items did not require knowledge beyond that given in the text itself. In Experiment 2, the same and additional conditions were included to test the importance to true/false performance of the extent to which situational and text representations are developed.

One of the ANOVAs reported earlier showed an effect of processing time on the recall measure. Those whose processing time was determined by the read-and-do subject in their set (yoked subjects) spent more time with the text $(M=3.73 \mathrm{~min})$ and showed better recall than did unyoked subjects, who spent less processing time $(M=$ $2.38 \mathrm{~min}$ ). Therefore, the recall comparisons were performed with the yoked and unyoked conditions separately. With exposure time controlled, the text representation theory led to the prediction of better recall performance in the read-and-see-yoked and read-only-yoked conditions, because the subjects in these conditions would have more time to study the text, which would allow them to develop a better textbase. On the other hand, dual coding theory led to the prediction that read-and-do subjects' recall performance should be best, because more codes lead to better recall. Figure 3 shows the theory predictions and the observed means for each condition. As predicted by the text representation theory, and contrary to dual coding, the read-and-see-yoked/readonly-yoked subjects $(M=15.29)$ showed better recall than did the read-and-do subjects $(M=11.61)[t(45)=$ $2.15, p<.05]$. There was no difference between the read-

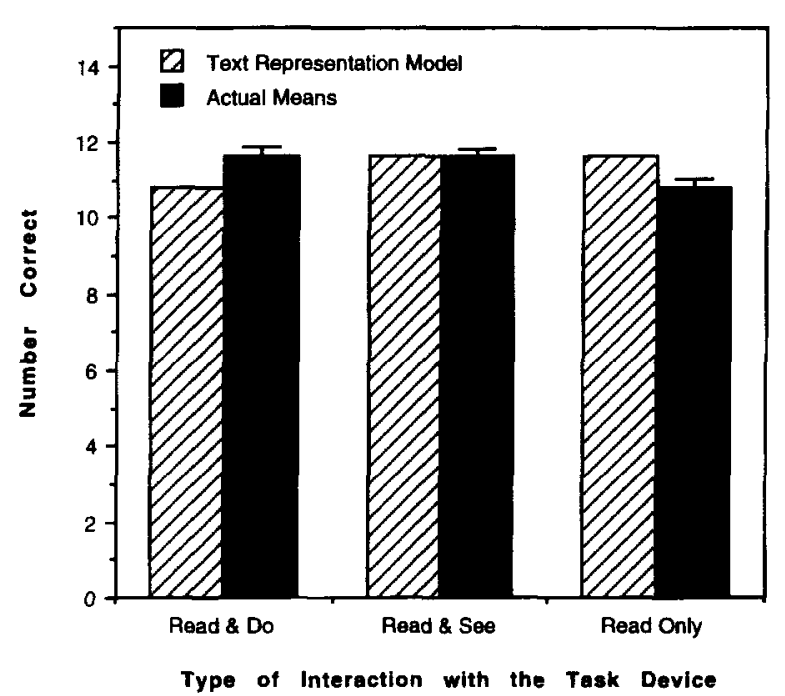

Figure 2. Text representation theory true/false predictions and observed means from Experiment 1.

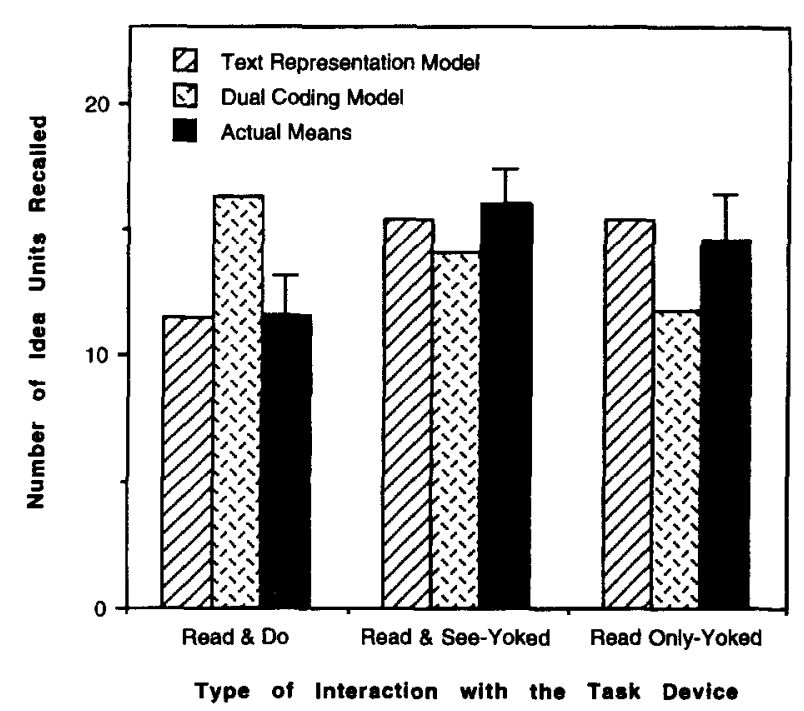

Figure 3. Recall predictions from the text representation and dual coding theories and observed means from Experiment 1.

and-see-unyoked/read-only-unyoked $(M \doteq 11.61)$ and read-and-do $(M=11.61)$ conditions. The text representation theory's predictions for the yoked conditions fit the data better than would be expected by chance $\left[\chi^{2}(2)=\right.$ $0.073, p<.05]$; however, dual coding's did not $\left[\chi^{2}(2)=\right.$ $2.26, p>.05]$.

The pattern of the recall means indicated that doing, rather than facilitating recall (as would be predicted by dual coding), might interfere by directing subjects' attention away from the text itself. The comparison of those who did the task while reading and those who had the same amount of time to read while only looking at the device or only reading suggested that a larger amount of text study time led to a better text representation, which, in turn, led to better recall. In addition, the difference between the performance of the yoked and unyoked subjects confirmed the importance of processing time on recall. The subjects who spent more time with the text recalled more than did those who spent less time. However, processing time had no effect on the true/false and task performance time measures.

\section{EXPERIMENT 2}

There is some ambiguity in the task performance results. Was performance better in the read-and-do condition than in the read-only condition simply because the subjects in the former condition had an opportunity to practice doing the task while reading? In order to get the observed facilitation, is it necessary to actually do the task, or would seeing someone else do the task or imagining oneseif doing it be enough? These questions were tested in Experiment 2 by including two additional conditions: read-and-see-experimenter-do (wherein the subjects saw the experimenter do the step-by-step task while reading but did not practice performing the task) and 
read-and-imagine (wherein the subjects imagined themselves doing each step of the task without access to the device).

The text representation theory predicts that the readand-do, read-and-see, and read-and-see-experimenter-do subjects will have good situational models and therefore will do well on the task performance measure, because they all have access to the device while reading. However, the read-and-do subjects should do best, because they will engage in transfer-appropriate processing. The read-and-see-experimenter-do subjects will perform second best, because they have an opportunity to observe task performance. The read-and-see subjects will perform more slowly, because they do not witness task performance. The read-only and read-and-imagine subjects' task performance will be equally poor, because they are limited to the information given in the text, and, therefore, their situational models will be poorly developed.

The text representation theory predicts that recall and true/false performance will be affected by the quality of the propositional representation. The read-only and read-and-see conditions will have well-developed textbases because the read-only subjects will have only the text on which to focus and the read-and-see subjects will look at the device while reading, which will not interfere with the development of a good text representation. In fact, it may improve it because they can better understand what they are reading. Therefore, read-only and read-and-see subjects will show the best true/false and recall performance. The textbases of the read-and-do, read-and-see-experimenter-do, and read-and-imagine conditions will be poorly developed, in part because they are task oriented, which will lead these subjects to focus less on the text. In addition, the read-and-imagine subjects' text representation will be poor because their incorrect situation model (developed by imagining without the device present) will interfere with their memory for the text. The read-and-see-experimenter-do subjects will see the task enacted. The situation will be made clear, leading them to pay less attention to the text. Therefore, the read-and-see-experimenter-do and read-and-imagine subjects will perform less well on the true/false and recall measures. The read-and-do subjects will show the worst recall and true/false performance, because the demands of doing the task while reading will give them less time to study the text.

In Experiment 2, we again tested the dual coding theory with the recall measure. From Paivio's (1991) theory, we assume that something experienced through the relevant sense organ (direct experience, e.g., for the development of a proprioceptive code, doing the task oneself) would lead to a stronger memory representation than would something experienced indirectly (e.g., watching someone perform the task). Imagining oneself doing the task would be an indirect and potentially incorrect proprioceptive experience. Therefore, the read-and-do subjects should have the best recall because the information is coded as verbal, direct visual, and direct proprioceptive. The read-and-see-experimenter-do subjects should be next best, with verbal, direct visual, and indirect proprioceptive codes. The read-and-see subjects will have coded the information verbally and visually direct. The read-and-imagine subjects will have a verbal code and indirect (and potentially incorrect) visual and proprioceptive codes. The read-only subjects should be worst, because the only code they will definitely have is verbal.

In Experiment 2, the number of subjects in each condition was increased in order to have more power to detect whether simply seeing the device is sufficient for the development of a solid text and task representation. Because yoking had minimal effects in Experiment 1 (except on recall), we eliminated this variable in Experiment 2 to make this experiment more manageable. In Experiment 2, we controlled for amount of text exposure-that is, subjects in all conditions spent a fixed amount of time with the texts.

\section{Method \\ Design}

Experiment 2 included the read-and-do, read-and-see, and readonly conditions from Experiment 1, except that, instead of subjects' text exposure time being yoked to that of a read-and-do subject, all participants were given a fixed amount of text exposure time ( $35 \mathrm{sec}$ for practice, $4 \mathrm{~min}$ for Spool, and $4.5 \mathrm{~min}$ for Clock). The times were based on the read-and-do times from Experiment 1 . Times were chosen so that 8 of the 10 read-and-do subjects in Experiment 1 used the selected time or less to read the text and do the task.

The two additional conditions in Experiment 2 were read-andsee-experimenter-do and read-and-imagine. In the read-andimagine condition, the subjects imagined each task step after they read about it; in the read-and-see-experimenter-do condition, the subjects watched the experimenter perform each step after they read it. As in Experiment 1, the dependent measures were task performance time, number correct on true/false, and number of idea units correctly recalled.

\section{Subjects}

A total of 85 psychology student volunteers (51 females and 34 males) from a Midwestern university were randomly assigned to one of five conditions, with the constraint that each condition had approximately the same proportion of males and females. Two of the read-and-do subjects were unable to complete the Spool task in the time allotted during reading, so their data were not used. The subjects participated in the experiment for extra credit in their psychology course.

\section{Materials}

The materials used were the same as those in Experiment 1 , with several exceptions. The Mechanical Movements Test and the Nelson-Denny Reading Test were not used. The "battery replacement" section of Clock was removed from the text. The texts were divided into steps for the second reading. Each step was typed onto a $3 \times 5$ in. index card. Each card began with a step (a procedure to be performed), with the exception of the first Spool card. The text continued on the card until the next step, which started the next card.

\section{Procedure}

In Experiment 2, the subjects were randomly assigned to one of the five conditions rather than being matched on mechanical or reading ability; therefore, they attended only one session.

For the subjects' first reading. the texts were typed on a sheet of paper in their entirety (as in Experiment 1). For the second reading, the text was divided into steps, and each step was placed on a 
separate index card because the subjects needed a clear idea of when to look at the experimenter in the read-and-see-experimenterdo condition during the second reading. In all conditions for the second reading, the subjects were instructed to read the card and then, depending on their condition, to perform the step (read-anddo), imagine the step (read-and-imagine), look at the device (readand-see), or watch the experimenter perform the step (read-andsee-experimenter-do). In the read-only condition, the subjects were instructed to simply read the card. As in Experiment 1, if the subjects had time after the second reading, they read over the text on cards for the remaining allotted time.

One other difference between the studies concerned the recall measure. In Experiment l, the subjects were instructed to recall the main ideas of the text; in Experiment 2, they were asked to try to "write down the text word for word." This was done in hopes of accentuating any differences between the conditions on the recal measure.

\section{Scoring}

Scoring was the same as that in Experiment 1.

Any scores that fell outside of 3 standard deviations were eliminated. This affected 2 subjects' performance time data in the readand-imagine condition and 1 each in the read-and-see and readand-see-experimenter-do conditions. One read-and-do subject's score on the true/false measure was also eliminated on the basis of this criterion.

The percent agreement on the recall scoring was $93 \%$ for both Spool and Clock

The amount of time it took the subjects to put the rubber band through the hole in the spool was removed from all subjects' data in this experiment, because the subjects were not provided with a wooden matchstick during the Spool task (as they were in Experiment 1). The matchstick was very helpful for pushing the rubber band through the hole in the spool. In order to make performance times comparable across the two experiments, the time it took to complete this step was subtracted from total performance time, on the basis of the videotaped recordings, by a judge who was blind to condition.

\section{Results and Discussion}

Prior to carrying out the comparisons of interest, we performed several analyses separately for each of the two texts to make sure the conditions did not vary in familiarity or first-reading time. ANOVAs found no difference between the conditions with respect to either how familiar the subjects were with the tasks $\left(F_{\mathbf{s}}<1\right)$ or the amount of time it took them to read the texts the first time $\left(F_{\mathrm{s}}<1\right)$.

As in Experiment 1 , for the following analyses only, we converted the recall and true/false scores to proportions (because there were different possible scores across the two texts) before we performed a three-way ANOVA. The between-subjects factors were text order and condition, and the within-subjects factor was text. There were main effects of text for both the recall measure [mean proportion for Clock $=.36$, and mean proportion for Spool $=$ $.26 ; F(1,73)=35.08, p<.001]$ and the performance time measure [Clock $M=1.96$, and Spool $M=1.44 ; F(1,69)$ $=11.88, p<.01]$. For the three dependent measures, there was no main effect of text order and there were no significant two- or three-way interactions, so we combined across order and text for the following comparisons. The means and standard deviations for each of the conditions, combined across text and order, are shown in Table 2.

The predicted and observed means for the performance time measure are shown in Figure 4. Although all of the observed means were not in the direction predicted by the text representation theory, the theory fit the data better than would be expected by chance $\left[\chi^{2}(4)=0.134\right.$, $p<.01]$. As predicted by the text representation theory, the read-and-imagine/read-only subjects $(M=2.05)$ performed the task more slowly than did the read-and-see subjects $(M=1.55)[t(74)=1.98, p<.05]$. Contrary to the text representation theory's predictions, the readand-see-experimenter-do mean was smallest, and the read-and-do and read-and-see means were very similar to each other.

Figure 5 shows the predicted and actual means for the true/false measure. The pattern of observed means was not completely consistent with what was predicted, but the theory's predictions fit the data better than would be expected by chance $\left[\chi^{2}(4)=0.031, p<.01\right]$. The readand-see/read-only $(M=11.50)$ versus read-and-seeexperimenter-do/read-and-imagine $(M=10.96)$ comparison was significant $[t(77)=2.30, p<.05]$. However, rather than being smaller, the read-and-do mean (11.00) was comparable to the read-and-see-experimenter-do/ read-and-imagine mean (10.96).

The opposing predictions of the dual coding and text representation theories for the recall data are shown in Figure 6 . The predicted means from the text representation theory fit the data very well $\left[\chi^{2}(4)=0.019, p<.01\right]$, whereas dual coding's did not $\left[\chi^{2}(4)=5.12, p>.05\right]$. As predicted by the text representation theory, the read-anddo subjects $(M=8.37)$ recalled less than did the readand-see-experimenter-do/read-and-imagine subjects $(M=11.26)[t(78)=1.83, p<.05]$. The read-and-seeexperimenter-do/read-and-imagine $(M=11.26)$ versus

Table 2

Means From Experiment 2 Averaged Across Text and Order

\begin{tabular}{|c|c|c|c|c|c|c|c|c|c|c|c|c|c|c|c|}
\hline \multirow{3}{*}{$\begin{array}{l}\text { Dependent } \\
\text { Measure }\end{array}$} & \multicolumn{15}{|c|}{ Condition } \\
\hline & \multicolumn{3}{|c|}{ Read-and-Do } & \multicolumn{3}{|c|}{ Read-and-See } & \multicolumn{3}{|c|}{$\begin{array}{l}\text { Read-and-See- } \\
\text { Experimenter-Do }\end{array}$} & \multicolumn{3}{|c|}{ Read-and-Imagine } & \multicolumn{3}{|c|}{ Read-Only } \\
\hline & $M$ & $S D$ & $n$ & $M$ & $S D$ & $n$ & $M$ & $S D$ & $n$ & $M$ & $S D$ & $n$ & $M$ & $S D$ & $n$ \\
\hline Performance time & 1.56 & 0.46 & 15 & 1.55 & 0.65 & 16 & 1.29 & 0.49 & 16 & 2.10 & 0.71 & 15 & 2.00 & 0.75 & 17 \\
\hline True/false & 11.00 & 0.55 & 14 & 11.79 & 0.77 & 17 & 11.03 & 0.89 & 17 & 10.88 & 1.43 & 17 & 11.21 & 0.95 & 17 \\
\hline Recall & 8.37 & 4.36 & 15 & 13.04 & 4.54 & 17 & 11.38 & 5.43 & 17 & 11.13 & 5.25 & 17 & 13.49 & 5.63 & 17 \\
\hline
\end{tabular}

Note-Performance times are given in minutes. True/false values are expressed in terms of the number of questions correctly answered. Recall values are expressed in terms of the number of idea units correct. 


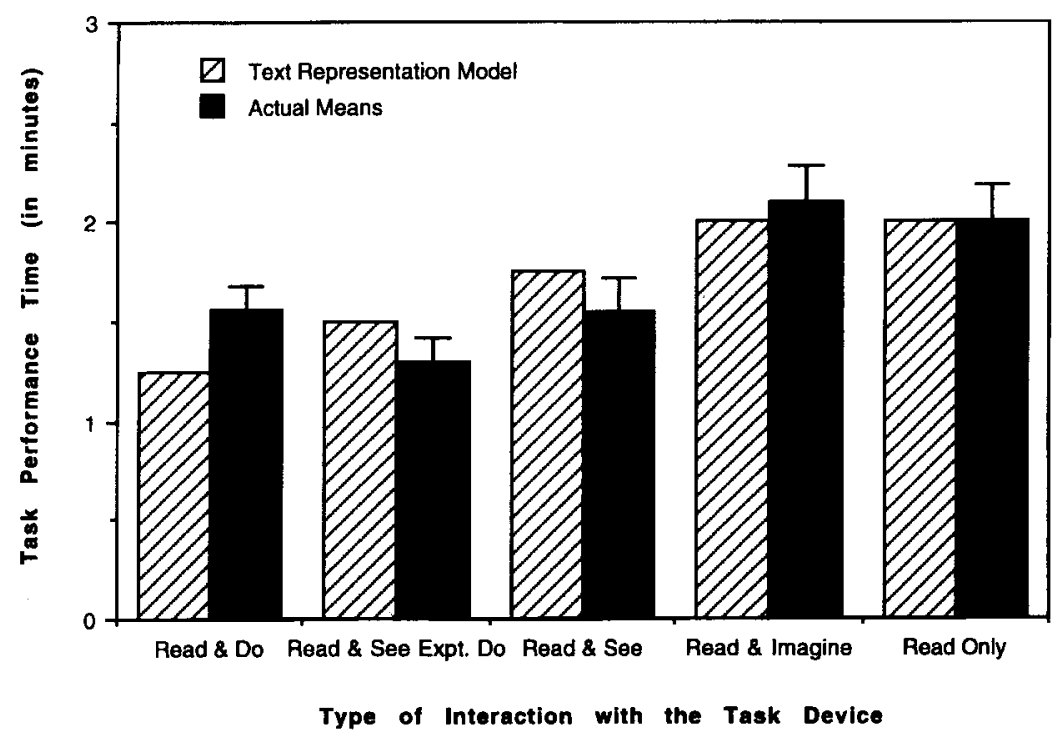

Figure 4. Text representation theory performance time predictions and observed means from Experiment 2.

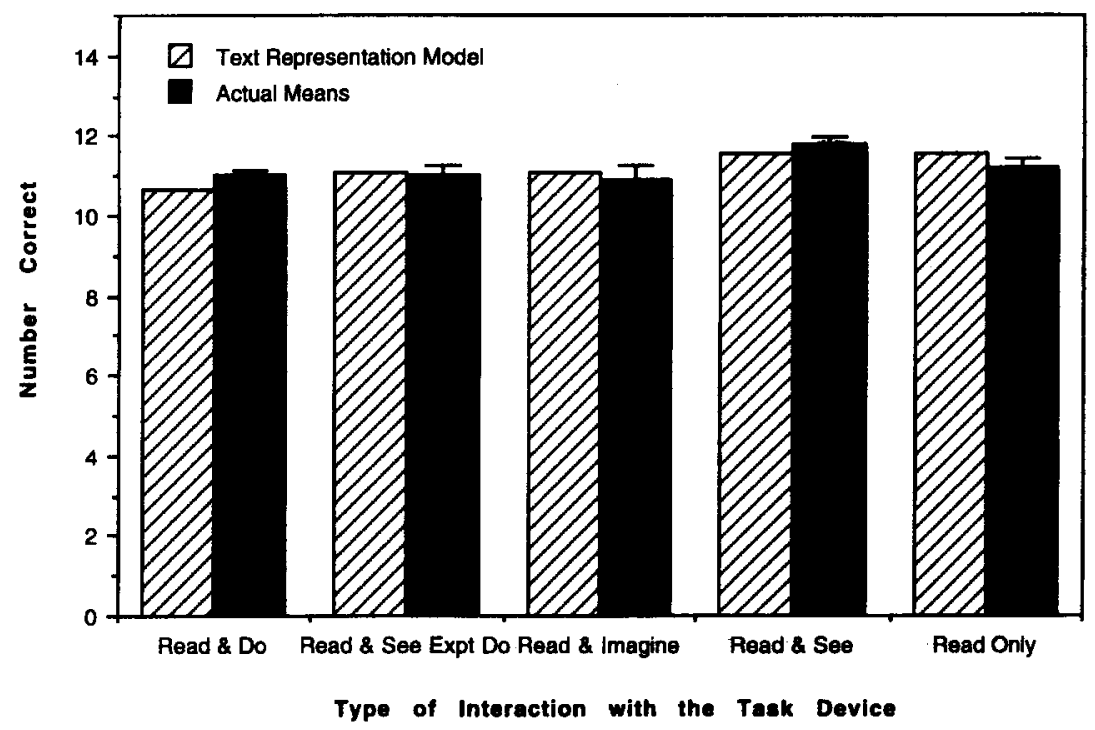

Figure 5. Text representation theory true/false predictions and observed means from Experiment 2.

read-and-see/read-only $(M=13.26)$ contrast was marginally significant $[t(78)=1.63, p=.054]$.

With each of the three dependent measures, the text representation theory fit the data better than would be expected by chance. Also, when the goodness of the two theories was compared for the recall measure, the text representation theory did a better job of predicting the data than did the dual coding theory.

As predicted by the text representation theory, the read-and-see-experimenter-do, read-and-do, and readand-see subjects performed the task faster than did the read-only and read-and-imagine subjects. This indicates that access to the device enables the development of a better situational representation, which leads to better task performance. With the recall, the read-only, readand-see-experimenter-do, and read-and-see subjects scored significantly higher than did the read-and-do subjects. The recall means of the read-only and read-and-see conditions were higher than those of the read-and-seeexperimenter-do and read-and-imagine conditions, but the comparison was marginally significant. These results suggest that a task orientation leads the reader to focus less on the text, leading to a poorer propositional representation. The combination of read-and-see and 


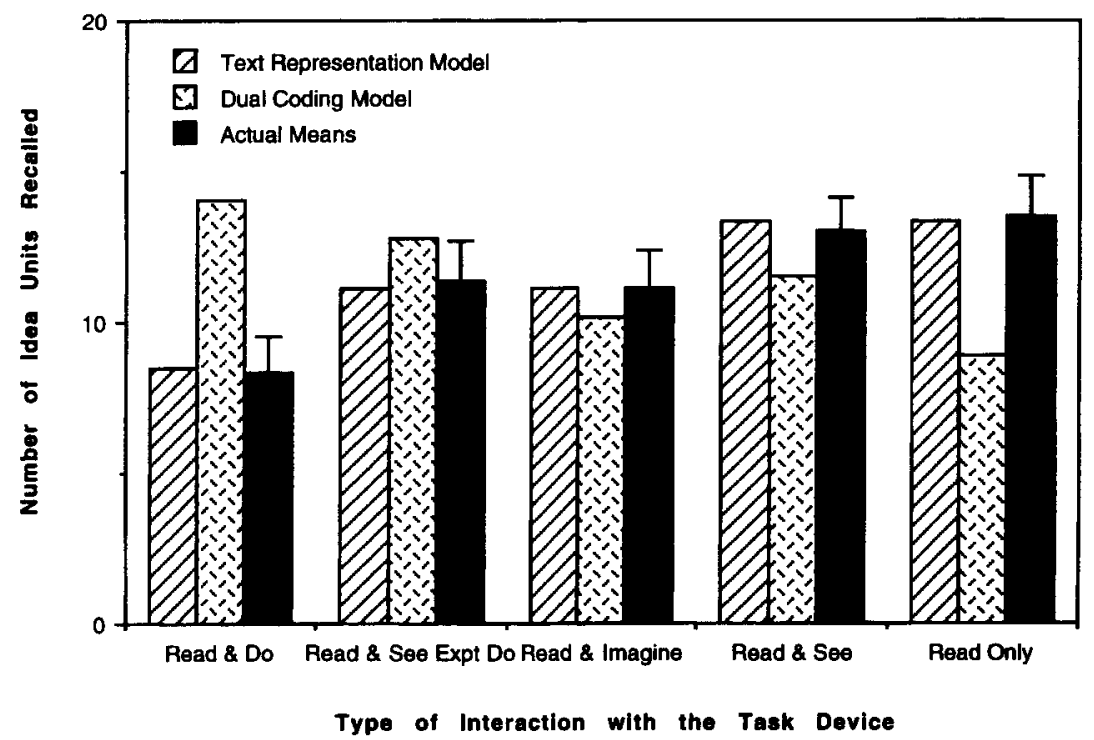

Figure 6. Recall predictions from the text representation and dual coding theories and observed means from Experiment 2.

read-only was better than the other conditions on true/ false. While both of these means were larger than those from the other conditions, the read-and-see mean was larger than the read-only mean and probably carried this contrast. The excellent performance of the read-and-see subjects on both recall and true/false is consistent with the notion that, under circumstances where the textbase is well established, a good situational representation can enhance memory for the text.

The results of Experiment 2 clarified the question of a practice effect for the read-and-do condition in two ways. First, with more power (from a larger sample), the means of the read-and-do and read-and-see conditions were nearly identical on the performance time measure, suggesting that, with these procedural texts, practicing the task does not lead to a better situational representation than does merely having the device available to look at while reading. Second, the read-and-do subjects did not perform better than did the read-and-see-experimenterdo subjects, which should have happened on the basis of transfer-appropriate processing.

\section{GENERAL DISCUSSION}

The text representation theory was supported across the two experiments. In the present experiments, the extent to which the subjects interacted with the task device had differential effects on their task performance times, true/false scores, and recall. From this, we infer that the different conditions of the experiments led to different mental representations of the task and the text. Doing the task, watching the experimenter do the task, or seeing the device described by the text while reading led to the formation of a better situational representation, resulting in better task performance than did imagining the task while reading or simply reading the text. Also consistent with this theory, it appeared that focusing on the task, and therefore not paying as much attention to the text, resulted in a poorer propositional representation. Therefore, there was better recall performance when the subjects only read the text or read while seeing the device than when they read the text while watching the experimenter do the task or imaging doing the task. These latter conditions, in turn, led to better recall than did reading while doing the task. The results from the true/false test were somewhat inconsistent across the two experiments, although, in both, reading while seeing the device led to very good performance on this task, which was predicted by the text representation theory. The pattern of results from these experiments extends the findings of Schmalhofer and Glavanov (1986), Mills et al. (1995), and Mannes and Kintsch (1987).

The information available when doing the task while reading, watching it being done, or only seeing the device enabled the reader to form an accurate mental model of the task. While many of the subjects had used a digital clock and had made homemade toys prior to their participation in the study, very few had directly comparable experience. Therefore, not having access to the device (as when imagining the task being performed or only reading) would likely lead to the formation of an inaccurate mental representation of the task. Reder, Charney, and Morgan (1986) have noted the importance of accurate text elaborations. Subject-generated elaborations will aid performance only if they fit with what is being read, and this depends on the subjects' situational knowledge. Without the information needed to accurately update their situational model, those who only read the text or read and imagined the task may have formed inappropriate elaborations, and these might have interfered with later performance (Reder et al., 1986). On the other hand, those who saw the experimenter per- 
form the task may have had an advantage on the task performance measure over all of the other conditions, because they saw the optimal way to do the task from the start. They did not have to figure out for themselves how to do the task. This allowed them to develop an accurate situational representation quickly and easily.

It is surprising that there was no practice effect for those who performed the task while reading. In Experiment 1 , although the difference was not significant, performance was better for those who had a chance to practice the task than for those who only saw the device. In Experiment 2, the means from these two conditions were almost identical. If those who only saw the device while reading performed better in Experiment 2 than they did in Experiment 1, it might be because of a procedural difference. In Experiment 1, the subjects who had access to the device while reading looked at it only when they chose to. In Experiment 2, for the second text reading, each step of the procedure was presented on a separate card, and the subjects who saw the device while reading were instructed to look at the device after they read each step. This division into steps may have made the procedural nature of the text more salient, and the "enforced" looking may have improved these subjects' situational representation. In turn, this improved situational representation may have resulted in a performance time that was comparable to the performance time of those who performed the task while reading. In a study in which subjects learned to tie a bowline knot with the help of prose and/or pictures, Hayes and Henk (1986) interviewed subjects after they had completed the experiment. They found that the prose-plus-pictures subjects who tied the knot successfully used the pictures to verify their interpretation of the text. The types of tasks used in the present study were such that all of the information necessary to confirm a text interpretation was available by looking at the device, and this may have minimized the importance of motoric enactment.

On the recall measure, doing the task interfered with the development of a propositional representation. The most likely explanation is that the subjects in the readand-do condition had little time to study the text. Generally speaking, doing the task took more time than did looking at the device, watching the experimenter do the task, or imagining doing the task. The pattern of the recall data, however, suggests that less time with the text cannot account for the poorer recall performance of those who read while watching the experimenter or read while imagining, relative to the performance of those who only read or read while looking at the device. In both of the former conditions, the subjects had additional time to study the text (and, particularly for those who read and imagined, comparable time to those who read while looking at the device). As noted earlier, seeing the experimenter do the task so easily may have given these subjects a false sense of confidence about the text, which may have caused them to neglect their textbase development. Those who read while imagining probably imag- ined some of the steps incorrectly, and this would have had a negative effect on recall.

The present recall results do not support early research conducted with simple procedures that used recall as the dependent measure (e.g., Cohen, 1981; Saltz \& Donnenwerth-Nolan, 1981). Unlike these earlier studies, in the present study performing the task did not lead to better recall than only reading the text. In fact, it led to poorer performance. There are many differences between the simple tasks used in the earlier studies and the long, multistepped tasks used in this research, which can help account for the different results. First, with simple tasks, subjects do not have to "figure out" what actions are required of them; tasks such as "crossing your fingers" or "flipping a pancake" do not require much thought. On the other hand, the tasks used in the present experiments did require some effort to figure out. Even though the objects used in the tasks were common and familiar to the subjects, how they were used or the relationship of the parts to each other had to be learned from the text. Second, the texts were obviously much more complicated. The simple procedures involved simple sentences that only had to be remembered. On the other hand, the texts used in this study involved integrating information across sentences, determining what was important and what was not. These differences between the two types of tasks and texts resulted in a much more cognitively demanding situation in the present experiments. Therefore, the same manipulation, motoric enactment of a procedure, resulted in very different findings. With the earlier simple tasks, motoric enactment allowed elaboration of the text information, which, in turn, improved recall. In the present study's yoked and timed conditions, enactment took away from study time. Presumably similar study time (in the unyoked conditions of Experiment 1) led to no difference in recall.

The present study provides more evidence that an adequate theory of procedural text comprehension and use must incorporate representations of the task (the situational representations). This may explain why some of the structural variables typically found to strongly influence reading performance with narratives are not so important with procedural texts (Mills et al., 1993). While structural variables are important to all text types, they are insufficient for predicting measures from procedural text. Some types of representations may be more important for some genres of text than others. Building an adequate situational representation may be more important for procedural text than for narratives; it is obviously critical when the goal of reading is task performance.

The results of this study make it clear that, when studying procedural text, it is probably not appropriate to use only a recall or comprehension measure. Usually, procedural text is read for the purpose of performing a task, not for recall or comprehension. In the present study, neither the recall nor the true/false scores mirrored the task performance results. For most research purposes, task performance is a more appropriate mea- 
sure for procedural text, even though it is much more difficult to collect than is recall or recognition.

The implications of these experiments for complex yet familiar procedural tasks of the type used in this work are: (1) Reading the text while seeing the device leads to the development of the best overall task and text knowledge. (2) Seeing someone else do the task is a good way to develop an accurate situational model quite easily, with less disruption to the development of a propositional representation (given the same amount of processing time) than is the case with reading while doing. (3) Imagining oneself doing the task while reading, without an adequate understanding of the task, does not appear to have any benefits over only reading the text on true/false and task performance, and it leads to poorer recall than does only reading. The results indicate that the extent to which one interacts with the device while reading affects the formation of different types of representations differently, and these representations, in turn, have different effects on a variety of measures.

\section{REFERENCES}

BäCKMAN, L. (1985). Further evidence for the lack of adult age differences on free recall of subject-performed tasks: The importance of motor action. Human Learning, 4, 79-87.

Blake, J., Austin, W., and Lowenstein, J. (1987). The effect of enactment upon sentence imitation in preschool children. Journal of Psycholinguistic Research, 16, 351-367.

Blaxton, T. A. (1989). Investigating dissociations among memory measures: Support for a transfer-appropriate processing framework Journal of Experimental Psychology: Learning, Memory, \& Cognition, 15, 657-668.

Brown, J. (1973). The Nelson-Denny reading test. Boston: Houghton Mifflin.

COHEN, R. L. (1981). On the generality of some memory laws. Scandinavian Journal of Psychology, 22, 267-281.

HAYES, D. A., \& HENK, W. A. (1986). Understanding and remembering complex prose augmented by analogic and pictorial illustration. Journal of Reading Behavior, 28, 63-78.

HerberT, D. (1980). Mr. Wizard's supermarket science. New York: Random House.

Kintsch, W. (1994). Text comprehension, memory, and learning. American Psychologist, 49, 294-303.

ManNeS, S. M., \& KinTSCH, W. (1987). Knowledge organization and text organization. Cognition \& Instruction, 4, 91-115.

McNamara, D. S., Kintsch, E., Songer, N. B., \& Kintsch, W. (in press). Are good texts always better? Interactions of text coherence, background knowledge, and levels of understanding in learning from text. Cognition \& instruction.

Mills, C. B., Diehl, V., Birkmire, D., Mou, L. C. (1993). Procedural text: Predictions of importance ratings and recall by models of reading comprehension. Discourse Processes, 16, 279-316.

Mills, C. B., Diehl, V., Birkmire, D., \& Mou, L. C. (1995). Reading procedural text: Effects of purpose and predictions of reading comprehension models. Discourse Processes, 20, 79-107.

PaIvio, A. (1991). Dual coding theory: Retrospect and current status. Canadian Journal of Psychology, 45, 255-287.

Perrig, W., \& Kintsch, W. (1985). Propositional and situational representations of text. Journal of Memory \& Language, 24, 503-518.

Reder, L. M., Charney, D. H., \& Morgan, K. I. (1986). The role of elaborations in learning a skill from an instructional text. Memory \& Cognition, 14, 64-78.

SAlTZ, E., \& Dixon, D. (1982). Let's pretend: The role of motoric imagery in memory for sentences and words. Journal of Experimental Child Psychology, 34, 77-92.
Saltz, E., \& Donnenwerth-Nolan, S. (1981). Does motoric imagery facilitate memory for sentences? A selective interference test. Journal of Verbal Learning \& Verbal Behavior, 20, 322-332.

SChmalhofer, F., \& Glavanov, D. (1986). Three components of understanding a programmer's manual: Verbatim, propositional, and situational representations. Journal of Memory \& Language, 25, 279-294.

Thurstone, L. L., \& JefFrey, T. E. (1984). Mechanical movements. Park Ridge, IL: London House.

VAN DiJK, T. A., \& KINTsCH, W. (1983). Strategies of discourse comprehension. New York: Academic Press.

\section{APPENDIX}

\section{How To Use A Digital Timer Clock}

To Read Time:

1. Normal display shows Hour:Minute.

2. Press D once to show Month \& Date.

3. Press D again to show Seconds only.

4. To return to normal display, press $\mathrm{D}$ once more.

To Set Time:

1. All setting is done by pressing $\mathrm{S}$. The setting sequence is: Month, Date, Hour, Minute, and Second.

2. To change any function, press $S$ until function to be changed appears. Press D until correct figure appears.

When all functions are correct, press $S$ until Hour:Minute shows with colon flashing. If colon is not flashing, press $\mathrm{D}$.

To Replace Battery:

1. Between case halves there is a gap where you can separate the unit into two parts.

2. Remove the two screws on the battery plate to remove this plate.

3. Turn over the plate and replace the used battery with a new one. Note: be sure to maintain correct polarity.

4. Reassemble the battery plate and body. Battery specification: one piece $1.5 \mathrm{~V} 23-115$ battery or equivalent. LR-44 or A76 may be used.

To Use Timer:

1. Press $T$ for timer mode. Display will show 0.00 .

2. Press $T$ again to start timing.

3. To stop timing, press $\mathrm{T}$ once more. Display will show elapsed time.

4. Press $D$ to return to normal display, or press $T$ again to reset timer.

5. Timer counts to 15 minutes, then resets to zero and keeps counting in 15-minute increments.

\section{How To Make And Use A Spool Vehicle}

A very long time ago someone figured out how to wind up a rubber band inside a spool and then make the rubber band unwind slowly to make the spool crawl across the floor.

A modern version uses a spool of any size. Slip a rubber band, which is about the same length as the spool, through the opening, so that it passes from one end of the spool to the other. Anchor one end of the rubber band with a tack to the end of the spool. Slip the other end of the rubber band through the hole in a metal washer. The washer creates enough friction to keep the rubber band from unwinding quickly and yet is slippery enough to allow the wheel to turn slowly. That's why the washer 
is called a slipper. If you don't have a washer, you can make a slipper from the plastic of a refrigerator container or coffee-can top.

Into the end of the rubber band and through the slipper, put the last part - the drag. It can be a match stick, knitting needle, pencil, and so on. If the rubber band is not taut, wind it several times around the drag to take up the slack.

To energize your spool vehicle, turn the drag to wind up the rubber band inside the spool. Set the spool down on the table. The drag keeps its end of the rubber band from turning. The twisting action of the rubber band is transferred via the anchor to the spool. With the right amount of friction from the slipper, the spool continues across the table until most of the energy you wound into the rubber band is released.

You can have races and battles if your friends also make spool vehicles.

(Manuscript received July 23, 1994;

revision accepted for publication January 24,1995 .) 